\title{
The prognostic predictors of patients with airway involvement due to advanced esophageal cancer after metallic airway stenting using flexible bronchoscopy
}

\author{
Wen-Chien Cheng ${ }^{1,2,3}$, Meng-Fang Shen ${ }^{1}$, Biing-Ru Wu ${ }^{1,2}$, Chih-Yu Chen ${ }^{1,2,3,4}$, Wei-Chun Chen ${ }^{1,2,3}$, \\ Wei-Chih Liao ${ }^{1,3,5}$, Chia-Hung Chen ${ }^{1,5,6}$, Chih-Yen Tu ${ }^{1,2,7}$ \\ ${ }^{1}$ Division of Pulmonary and Critical Care Medicine, Department of Internal Medicine, China Medical University Hospital, Taichung; ${ }^{2}$ School \\ of Medicine, ${ }^{3}$ Department of Internal Medicine, Hyperbaric Oxygen Therapy Center, ${ }^{4}$ Department of Respiratory Therapy, ${ }^{5}$ Graduate Institute \\ of Clinical Medical Science, China Medical University, Taichung; ${ }^{6}$ Taiwan Clinical Trial Consortium for Lung Diseases (TCoC), Taichung; \\ ${ }^{7}$ Department of Life Science, National Chung Hsing University, Taichung \\ Contributions: (I) Conception and design: WC Cheng, CH Chen, CY Tu; (II) Administrative support: WC Chen, WC Liao; (III) Provision of study \\ materials or patients: WC Liao, CH Chen; (IV) Collection and assembly of data: WC Cheng, MF Shen; (V) Data analysis and interpretation: WC \\ Chen, CH Chen;(VI) Manuscript writing: All authors; (VII) Final approval of manuscript: All authors. \\ Correspondence to: Chih-Yen Tu, MD; Chia-Hung Chen, MD. Division of Pulmonary and Critical Care Medicine, Department of Internal Medicine, \\ China Medical University Hospital, No. 2 Yude Road, Taichung. Email: chesttu@gmail.com; hsnu758@gmail.com.
}

Background: Patients with advanced esophageal cancer and airway involvement have a poor prognosis. Self-expandable metallic stent (SEMS) implantation via bronchoscopy can immediately relieve airway stenosis and improve survival. The purpose of this study was to determine the factors that predict survival in patients with airway involvement due to advanced esophageal cancer after SEMS implantation.

Methods: We conducted this retrospective study from February 2007 to October 2013 at a university hospital. Forty-two patients with advanced esophageal cancer and airway involvement were included. The patients underwent flexible bronchoscopy with electrosurgery and SEMS implantation under bronchoscopic visualization and local anesthesia with no fluoroscopic guidance throughout the procedure.

Results: Acute respiratory failure (ARF) occurred in 14 patients (33.3\%). After SEMS implantation, 28 patients $(66.7 \%)$ received additional anti-cancer therapy. The median survival after the procedure was 83 days. A longer survival was seen in the patients who received further anti-cancer therapy [hazard ratio (HR), 0.146; 95\% confidence interval (CI), 0.06-0.34; $\mathrm{P}<0.001]$. Persistent pneumonia and poor performance status (PS) were potential factors for not receiving further therapy. The patients who received anti-cancer therapy before the procedure (HR, 3.429; 95\% CI, 1.54-7.60; $\mathrm{P}=0.002)$ and those with ARF (HR, 5.224; 95\% CI, 2.23-12.26; $\mathrm{P}<0.001)$ had worse survival.

Conclusions: SEMS insertion with flexible bronchoscopy without fluoroscopic guidance in the patients with airway involvement due to advanced esophageal cancer was safe and feasible. The patients who received anti-cancer therapy before the procedure and those with ARF had a poor prognosis. Post-airway stenting therapy had the positive impact on survival in these patients.

Keywords: Esophageal cancer; airway invasion; stent; flexible bronchoscopy

Submitted Mar 18, 2019. Accepted for publication Aug 16, 2019.

doi: $10.21037 /$ jtd.2019.08.108

View this article at: http://dx.doi.org/10.21037/jtd.2019.08.108

(c) Journal of Thoracic Disease. All rights reserved. 


\section{Introduction}

Esophageal cancer with airway involvement is always advanced, inoperable, and metastatic, and is associated with a poor prognosis (1). Airway complications may be due to external compression or direct invasion by esophageal cancer. Progression to tracheoesophageal fistula (TEF) or central airway obstruction (CAO) can lead to a rapid decline in health status and acute respiratory failure (ARF) $(2,3)$. Interventional bronchoscopy with metallic airway stenting can relieve malignant CAO immediately and maintain lumen patency, thereby improving quality and functional status (4-8). Covered self-expandable metal stents (SEMSs) can be used to treat airway stenosis and seal off TEFs $(9,10)$, which can facilitate liberation from mechanical ventilation (MV) (11). In addition, bronchoscopic interventions also offer the opportunity of additional therapy for inoperable patients with malignant $\mathrm{CAO}$ after improving their performance status (PS), resulting in longer survival $(12,13)$.

The clinical factors associated with the best outcomes after airway stenting have yet to be determined. Several studies have indicated that additional therapy, PS, location of the fistula, prolonged pneumonia, and the site of stent placement may be independent factors influencing the survival of patients with TEFs secondary to esophageal and lung cancer after successful airway stent implantation $(13,14)$. In addition, previous studies have conducted analysis without classification according to the primary malignancy site and limited to TEFs. However, few studies have investigated the prognostic factors influencing the survival of patients with advanced esophageal cancer and airway involvement after airway stenting. Therefore, the aim of this study was to identify the prognostic factors of patients with advanced esophageal cancer with all forms of airway involvement including external compression, direct airway invasion, and TEFs after interventional therapeutic bronchoscopy with airway stent insertion.

\section{Methods}

\section{Enrolled patients}

This study was approved by the China Medical University Hospital Internal Review Board (DMR98-IRB-335), and the requirement for informed consent was waived. Over a period of 6 years (from February 2007 to October 2013), flexible bronchoscopy with metallic stents was performed in 42 patients with airway involvement due to advanced esophageal cancer. Informed consent was obtained from each patient and/or their family prior to this procedure. We retrospectively reviewed hospital records and procedure notes in order to extract the following data: age, gender, underlying disease, symptoms caused by esophageal cancer, bronchoscopic appearance (presence of intraluminal disease, extrinsic compression, or fistula), PS before the bronchoscopic procedure, stenting, modalities of anticancer treatment, and occurrence of procedure-related complications.

\section{Bronchoscopic procedure}

The initial symptoms before the procedure included dyspnea, cough, dysphagia, persistent aspiration pneumonia, and ARF. Flexible bronchoscopic interventions without fluoroscopic guidance were performed in bronchoscopy room intensive care units under local anesthesia with $2 \%$ xylocaine solution and consciousness sedation with intravenous midazolam $(5 \mathrm{mg})$. Ultraflex covered SEMSs (Boston Scientific, Natick, MA, USA) were implanted alone or after endobronchial electrosurgery based on the condition of the airway. Each patient underwent flexible bronchoscopy (Olympus BF-260; Olympus, Tokyo, Japan) through an endotracheal tube if they had ARF or via the oral cavity if they did not have ARF. The bronchoscope was then navigated to the proximal end of the lesion. A guidewire was inserted through the bronchoscopic channel and passed through the lesion site, and the scope was then removed. At this time, the endotracheal tube balloon was deflated, which allowed the bronchoscopy to pass through vocal cords easily. The bronchoscope was then reintroduced into the trachea through a nasal guard into the space between the tracheal wall and the endotracheal tube to inspect the location of the guidewire, and it was positioned next to the guidewire. Under direct bronchoscopic visualization, a delivery catheter (Boston Scientific, Natick, MA, USA) was advanced over the guidewire to release the stent. The delivery catheter and guidewire were then withdrawn, leaving the bronchoscope to check the position of the stent. If stent re-positioning was required, biopsy forceps (FB15C-1; Olympus, Tokyo, Japan) were used to hold the end of the stent, and a thread was passed through the last loop of the meshwork. By smoothly pulling or pushing the thread, we could adjust the position of the stent. During the procedure of stent insertion, $100 \%$ oxygenation and assistant/control mode-ventilator support with $0 \mathrm{cmH}_{2} \mathrm{O}$ positive end expiratory pressure (PEEP) level were given to the patients with ARF, and oxygenation was tapered to 


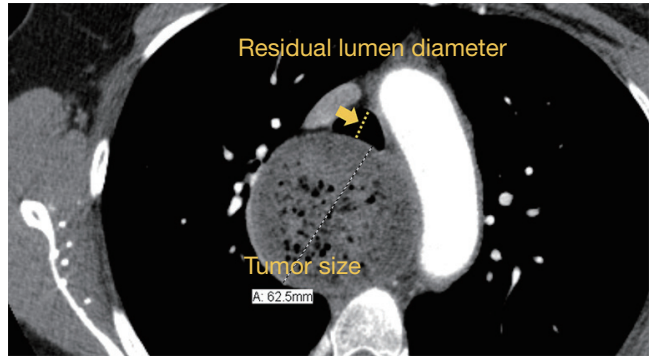

Figure 1 The esophageal tumor size was measured as the largest transverse width, and the residual diameter of an obstructed trachea was measured as the narrowest transverse width. The arrow to indicate where the "residual lumen diameter" is pointing.

less than $35 \%$ when electrocautery was performed. Oxygen supplementation with a nasal cannula or simple mask was also given to the patients without ARF. All of the patients underwent pulse oximeter and electrocardiogram (EKG) monitoring.

\section{Definitions}

The location of airway involvement was defined as the trachea, right main bronchus (RMB), left main bronchus (LMB), and multiple sites. The type of airway involvement was classified as external compression, invasion, fistula formation, and mixed (combination) (15). Persistent pneumonia was defined as persistent symptoms and radiological changes for 6 weeks or more despite treatment (16). The esophageal tumor size was measured as the largest transverse width, and the residual diameter of an obstructed trachea was measured as the narrowest transverse width (Figure 1). The definition of ARF is that the absent of ventilation and insufficient to maintain $\mathrm{O}_{2}$ intake and $\mathrm{CO}_{2}$ clearance, which required intubation and MV.

\section{Statistical analysis}

The data were compiled and analyzed using commercial statistical software MedCalc version 15.6.1 (MedCalc, Mariakerke, Belgium). All continuous variables were reported as median and interquartile range (IQR; $25^{\text {th }}$ and $75^{\text {th }}$ percentiles). Differences in continuous variables were compared using the Mann-Whitney U test. Categorical variables were reported as the number of patients and percentages. Differences in categorical variables were examined using Fisher's exact test. Univariate and multivariate Cox proportional hazards models were used to identify the independent prognostic factors for overall survival after airway stenting with flexible bronchoscopy. Differences in survival were analyzed using KaplanMeier log-rank analysis. The cut-off point of the residual diameter of obstructed airways in the patients with ARF was determined using receiver operating characteristic (ROC) curve analysis. All tests of significance were two sided, and a $\mathrm{P}$ value $\leq 0.05$ was considered to be statistically significant.

\section{Results}

The baseline clinical characteristics of the 42 patients with advanced esophageal cancer (17 stage III and 25 stage IV) with airway invasion who underwent airway stenting are summarized in Table 1. There were 39 males (92.9\%) with a median age of 53.5 years. Chronic obstructive pulmonary disease (COPD) was the most common comorbidity among the enrolled 42 patients. The average body weight (BW) and serum albumin level of the patients were $45.8 \mathrm{~kg}$ and $2.8 \mathrm{mg} / \mathrm{dL}$, respectively. Sixteen patients $(38.1 \%)$ had not previously undergone anti-cancer treatment, and 26 patients (61.9\%) had previously undergone anti-cancer treatment, of whom 23 received concurrent chemoradiotherapy (CCRT) and three received radiotherapy (RT) alone. The indications for interventional bronchoscopy in these patients were as follows: 14 patients (33.3\%) had ARF, 10 (23.8\%) had dyspnea, 8 (19.0\%) had recurrent aspiration pneumonia, $8(19.0 \%)$ had a persistent cough, and $2(4.8 \%)$ had dysphagia. The tumor size was significantly larger in those with ARF $(4.6 \mathrm{~cm})$ than in those without ARF $(3.7 \mathrm{~cm})$ $(\mathrm{P}=0.033)$. The residual diameter of obstructed airways was significantly smaller in those with ARF $(0.6 \mathrm{~cm})$ than in those without ARF $(1.34 \mathrm{~cm})(\mathrm{P}<0.001)$ (Table 2). The optimal cut-off value determined according to ROC analysis was $<0.77 \mathrm{~cm}$ (Figure 2). The patients with a residual diameter $<0.77 \mathrm{~cm}$ had a high risk of ARF. The occurrence of persistent pneumonia was significantly higher in those with $\mathrm{ARF}(9 / 14,64.3 \%)$ than in those without ARF $(8 / 28$, $28.6 \%)(\mathrm{P}=0.044)$. Before the procedure, $16(38.1 \%)$ and $18(42.9 \%)$ patients had a PS of 1 and 2, respectively, and 8 $(19.0 \%)$ patients had a poor PS (3 and 4).

Esophageal cancer was located predominantly in the upper to middle third of the esophagus ( $n=39,92.9 \%)$. The average size of the esophageal cancer was $4.02 \mathrm{~cm}$, and the average residual diameter of an obstructed airway was $0.83 \mathrm{~cm}$. Twenty-seven $(64.3 \%)$ patients had only tracheal involvement, 6 (14.3\%) had only LMB involvement, and the other $9(21.4 \%)$ patients had multiple site involvement (two 
Table 1 Baseline clinical characteristics of the 42 patients with advanced esophageal cancer with airway invasion

\begin{tabular}{|c|c|}
\hline Variables & No. (\%) or median (IQR) \\
\hline Age, year & $53.5[47-58]$ \\
\hline Male & $39(92.9)$ \\
\hline \multicolumn{2}{|l|}{ Esophagus status } \\
\hline Stage III & $17(40.5)$ \\
\hline Stage IV & $25(59.5)$ \\
\hline \multicolumn{2}{|l|}{ Underlying disease } \\
\hline HTN & $2(4.8)$ \\
\hline T2DM & $2(4.8)$ \\
\hline COPD & $5(11.9)$ \\
\hline Liver cirrhosis & $4(9.5)$ \\
\hline CVA & $1(2.4)$ \\
\hline $\mathrm{BW}, \mathrm{kg}$ & $45.8(41.5-55.7)$ \\
\hline Albumin, mg/dL & $2.80(2.5-3.2)$ \\
\hline \multicolumn{2}{|l|}{ Indication } \\
\hline Aspiration pneumonia & $8(19.0)$ \\
\hline Dyspnea & $10(23.8)$ \\
\hline ARF & $14(33.3)$ \\
\hline Cough & $8(19.0)$ \\
\hline Dysphagia & $2(4.8)$ \\
\hline \multicolumn{2}{|l|}{ Pre-stent treatment } \\
\hline Present/absent & $26(61.9) / 16(38.1)$ \\
\hline CCRT & $23(54.8)$ \\
\hline CT & $0(0)$ \\
\hline RT & $3(7.1)$ \\
\hline \multicolumn{2}{|l|}{ PS } \\
\hline 1 & $16(38.1)$ \\
\hline 2 & $18(42.9)$ \\
\hline 3 & $4(9.5)$ \\
\hline 4 & $4(9.5)$ \\
\hline
\end{tabular}

IQR, interquartile range; HTN, hypertension; T2DM, type 2 diabetic mellitus; COPD, chronic obstructive pulmonary disease; CVA, cerebrovascular accident; BW, body weight; ARF, acute respiratory failure; CCRT, concurrent chemoradiotherapy; CT, chemotherapy; RT, radiotherapy.

trachea and $\mathrm{RMB}$, five trachea and $\mathrm{LMB}$, one $\mathrm{RMB}$ and one $\mathrm{LMB}$, and one trachea, $\mathrm{RMB}$ and $\mathrm{LMB})$. With regards to airway involvement, 4 (9.5\%) patients had tracheal- esophagus fistulas alone, 4 (9.5\%) had only airway invasion caused by esophageal cancer, and 2 (4.8\%) had only airway external compression caused by esophageal cancer. Thirtytwo $(76.2 \%)$ patients had mixed types of airway involvement caused by esophageal cancer (Table 3).

The median survival period after airway stenting with flexible bronchoscopy was 83 days (IQR, 45-137 days). Twenty-eight patients $(66.7 \%)$ received additional therapy after airway interventions. Thirteen $(31.0 \%)$ patients received CCRT, 4 (9.5\%) received chemotherapy (CT), and $11(26.2 \%)$ received RT. Two patients underwent stent removal after the response to the post-procedural therapy. One received CT and the other received RT. The survival of patients after stent implantation were 71 days and 280 days, respectively. Fourteen (33.3\%) patients could not receive further therapy, of whom nine had persistent pneumonia and five had a poor PS (Table 4). Significantly more of the patients who did not receive further treatment had persistent pneumonia (9 of $14,64.3 \%$ ) compared to those who did receive further treatment ( 8 of $28,28.6 \%$ ) $(\mathrm{P}=0.044)$ (Table 5). Overall stent-related complications occurred in $22(52.4 \%)$ patients. Fifteen patients had a mucus plug, which was successfully relieved by washing the stent and bronchoscopy suction. Three (7.1\%) patients had granulation tissue formation at the ends of the stent which was successfully removed with forceps. Tumor ingrowth occurred in 16 patients, indicating disease progression. Procedure related complications occurred in 6 patients $(14.3 \%)$. Five patients had mild bleeding episode and resolved after the instillation of epinephrine (2 mL of 1:1,000 epinephrine mixed with normal saline in a 1:10 mixture). One patient had pneumothorax during bronchoscopy and resolved after pig tail insertion. The choice of additional treatment including CCRT, RT, and CT depended on the patient's PS and the decision of their physician.

The potential prognostic predictors after airway stenting are shown in Figures 3,4. The patients with PS $\leq 2$ had a longer survival (91 days) than those with PS $>2(50.5$ days) $(\mathrm{P}=0.038)$. A longer survival was also seen in the patients who received additional therapy (113.5 days) after airway stenting than those without further therapy $(36.5$ days $)(\mathrm{P}<0.001)$. The patients with mixed type airway involvement seemed to have a shorter survival (72 days) than those without mixed type airway involvement (172.5 days) $(\mathrm{P}=0.015)$. In addition, the patients with persistent pneumonia (51 days) also had a worse prognosis than those without persistent pneumonia (134 days) 
Table 2 Clinical characteristics of the 42 patients with/without ARF

\begin{tabular}{lccc}
\hline Variables & ARF $(n=14)$ & No ARF $(n=28)$ & P value \\
\hline Residual lumen diameter, cm & $0.6(0.55-0.70)$ & $1.34(0.93-1.66)$ & $<.001$ \\
Tumor size, cm & $4.6(3.77-5.92)$ & $3.7(3.10-4.31)$ & 0.033 \\
Persistent pneumonia & $9(64.3 \%)$ & $8(28.6 \%)$ & 0.044 \\
Mixed type & $12(85.7 \%)$ & $20(71.4 \%)$ & 0.450 \\
Multiple & $2(14.3 \%)$ & $7(25.0 \%)$ & 0.692 \\
\hline
\end{tabular}

Data were presented as median and IQR $\left(25^{\text {th }}\right.$ and $75^{\text {th }}$ percentiles). ARF, Acute respiratory failure; IQR, interquartile range.

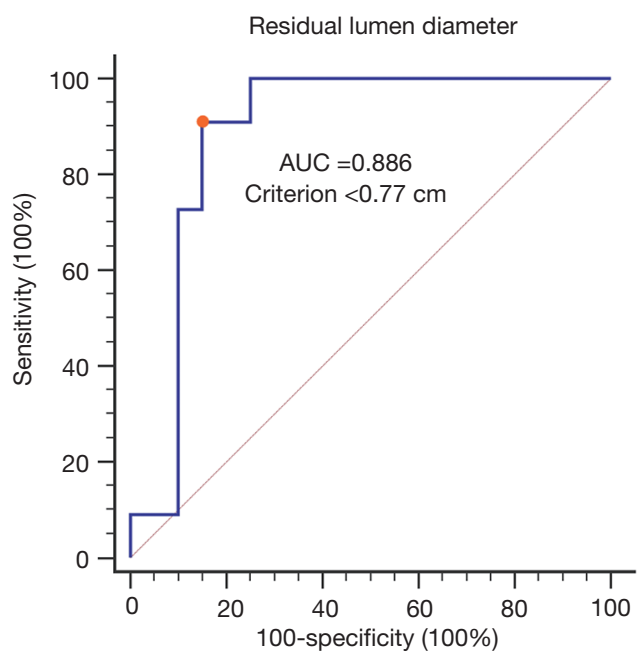

Figure 2 The optimal cut-off value of residual airway lumen diameter determined according to ROC analysis was $<0.77 \mathrm{~cm}$. The patients with a residual diameter $<0.77 \mathrm{~cm}$ had a high risk of ARF. AUC, area under the curve; ROC, receiver operating characteristic; ARF, acute respiratory failure.

$(\mathrm{P}<0.001)$. A shorter survival was also observed in the patients with ARF (55 days) compared to those without $\operatorname{ARF}(92.5$ days $)(\mathrm{P}=0.064)$.

Univariate analysis using a Cox proportional hazards model (Table 6) and Kaplan-Meier log-rank analysis (Figures 5,6) were performed with all variables that may be related to survival. ARF, additional therapy after airway stenting, mixed type airway involvement, PS, and persistent pneumonia were potential factors influencing the prognosis after airway stenting. Persistent pneumonia and PS $>2$ were not included in multivariate analysis due to the small number of patients $(n=42)$, and they were positively or negatively correlated with ARF and additional therapy after the procedure. We excluded the two variables in multivariate analysis to avoid multicollinearity. After multivariate
Table 3 Characteristics of the patients with advanced esophageal cancer with airway involvement

\begin{tabular}{|c|c|}
\hline Variables & No. (\%) or median (IQR) \\
\hline \multicolumn{2}{|l|}{ Position } \\
\hline $\mathrm{U} / 3$ & $18(42.9)$ \\
\hline$M / 3$ & $21(50.0)$ \\
\hline $\mathrm{L} / 3$ & $3(7.1)$ \\
\hline \multicolumn{2}{|l|}{ Tumor invasion position } \\
\hline Trachea & $27(64.3)$ \\
\hline RMB & $0(0)$ \\
\hline LMB & $6(14.3)$ \\
\hline Multiple & $9(21.4)$ \\
\hline$T+R$ & $2(4.8)$ \\
\hline$T+L$ & $5(11.9)$ \\
\hline$R+L$ & $1(2.4)$ \\
\hline$T+R+L$ & $1(2.4)$ \\
\hline Tumor size, $\mathrm{cm}$ & $4.02(3.2-5.0)$ \\
\hline The residual lumen diameter, $\mathrm{cm}$ & $0.83(0.60-1.57)$ \\
\hline \multicolumn{2}{|l|}{ The way of airway involvement } \\
\hline TEF & $4(9.5)$ \\
\hline Invasion & $4(9.5)$ \\
\hline External compression & $2(4.8)$ \\
\hline Mixed & $32(76.2)$ \\
\hline TEF + invasion + compression & $9(21.4)$ \\
\hline TEF + invasion & $3(7.1)$ \\
\hline TEF + compression & $3(7.1)$ \\
\hline Invasion + compression & $17(40.5)$ \\
\hline
\end{tabular}

$I Q R$, interquartile range; $U / 3$, upper third; $M / 3$, middle third; $L / 3$, lower third; RMB, right main bronchus; LMB, left main bronchus; $\mathrm{T}$, trachea; $\mathrm{R}$, right main bronchus; $\mathrm{L}$, left main bronchus; TEF, tracheoesophageal fistula. 
Table 4 Outcomes of airway stenting by flexible bronchoscopy

\begin{tabular}{|c|c|}
\hline Variables & No. (\%) or median (IQR) \\
\hline Survival days after stenting, day & $83[45-137]$ \\
\hline Diagnosis to stent place, day & $40.5[12-235]$ \\
\hline \multicolumn{2}{|l|}{ Stent placement } \\
\hline Single & 35 (83.3) \\
\hline Double & $7(16.7)$ \\
\hline \multicolumn{2}{|l|}{ Post-stent treatment } \\
\hline Present & $28(66.7)$ \\
\hline CCRT & $13(31.0)$ \\
\hline CT & $4(9.5)$ \\
\hline RT & $11(26.2)$ \\
\hline Stent related complications & $22(52.3)$ \\
\hline Mucus plug & $15(35.7)$ \\
\hline Granulation tissue & $3(7.1)$ \\
\hline Tumor ingrowth & $16(38.1)$ \\
\hline Procedure related complications & $6(14.3)$ \\
\hline Pneumothorax & $1(2.4)$ \\
\hline Mild bleeding & $5(11.9)$ \\
\hline Persistent pneumonia & $17(40.5)$ \\
\hline No post-stent treatment & $14(33.3)$ \\
\hline Persistent pneumonia & $9(21.4)$ \\
\hline Poor PS & 5 (11.9) \\
\hline \multicolumn{2}{|l|}{ The cause of death } \\
\hline Pneumonia & $14(33.3)$ \\
\hline Disease progression & $20(47.6)$ \\
\hline Massive bleeding & $2(4.8)$ \\
\hline Unknown cause & $6(14.3)$ \\
\hline
\end{tabular}

Data were presented as median and IQR $\left(25^{\text {th }}\right.$ and $75^{\text {th }}$ percentiles). IQR, interquartile range; CCRT, concurrent chemoradiotherapy; CT, chemotherapy; RT, radiotherapy; PS, performance status.

analysis, a longer survival was found in the patients who received additional therapy after airway stenting [hazard ratio (HR), 0.146; 95\% confidence interval (CI), 0.06-0.34; $\mathrm{P}<0.001]$. The patients who received previous anti-cancer treatment (HR, 3.429; 95\% CI, 1.54-7.60; $\mathrm{P}=0.002)$ and those with ARF (HR, 5.224; 95\% CI, 2.23-12.26; $\mathrm{P}<0.001$ ) before the procedure had a shorter survival.

\section{Discussion}

In this study, we demonstrated that airway stenting through flexible bronchoscopy without fluoroscopic guidance in patients with advanced esophageal cancer and airway involvement was safe and feasible. It offered the opportunity of additional therapy after interventional bronchoscopy, which had positive impact on survival. Persistent pneumonia and poor PS were the main reasons for not receiving further therapy. The patients who had received anti-cancer treatment and those with ARF before the procedure had a poor prognosis. ARF may have been related to the size of the esophageal cancer tumors and residual lumen diameter, as the patients with a residual lumen diameter $<0.77 \mathrm{~cm}$ had a high risk of ARF.

It is well known to be associated with improved survival in patients with malignant CAO or TEFs who receive additional therapy after airway stent implantation $(7,13,14,17,18)$. Our study results consistently showed that further additional therapy after airway stent implantation significantly prolonged survival (113.5 vs. 36.5 days). Previous studies have been limited to TEF $(13,14)$ and included primary airway tumors $(7,14,18)$, and the primary tumors themselves could be an influencing factor for the survival of these patients. McGrath et al. reported that the mean survival time of stented non-small cell lung cancer (NSCLC) was longer than that for esophageal cancer (214 vs. 70 days) (19). Patients with primary airway tumors have also been reported to have a better prognosis than those with other solid tumors with airway involvement (20). Therefore, our results offer more precise information for clinicians in the treatment of advanced esophageal cancer with all forms of airway involvement before and after bronchoscopy interventions.

Our study showed that the patients who received previous anti-cancer treatment on admission had a worse prognosis than those who did not receive treatment (68.5 vs. 90 days), which is consistent with previous studies $(12,18)$. In multivariate analysis, treatment-naïve status was an independent good prognostic predictor after airway stenting. The patients who received pre-procedure anticancer therapy may have had a poorer PS after cancer treatment and progressive disease status, which may have led to a worse prognosis despite additional therapy and longer survival than those with $\mathrm{PF}>2$ (91 vs. 50.5 days). Nagano et al. also reported that airway stenting may be more effective for patients with CAO due to advanced cancer with a good PS than those with a poor PS (21). 
Table 5 Clinical characteristics of the 42 patients with/without further anti-cancer treatment after airway stent implantation

\begin{tabular}{lccc}
\hline Variables & With further treatment $(\mathrm{n}=28)$ & Without further treatment $(\mathrm{n}=14)$ & $\mathrm{P}$ value \\
\hline Age, year & $54[38-70]$ & $52[42-79]$ & 0.925 \\
BW, kg & $47[34-51]$ & $43[34-76]$ & 0.151 \\
Albumin, $\mathrm{mg} / \mathrm{dL}$ & $3.0(2.48-3.60)$ & $2.8(2.5-3.0)$ & 0.209 \\
PS $>2$ & $3(19.0 \%)$ & $5(33.3 \%)$ & 0.092 \\
Persistent pneumonia & $8(28.6 \%)$ & $9(64.3 \%)$ & 0.044 \\
\hline
\end{tabular}

Data were presented as median and IQR $\left(25^{\text {th }}\right.$ and $75^{\text {th }}$ percentiles). BW, body weight; PS, performance status; IQR, interquartile range.
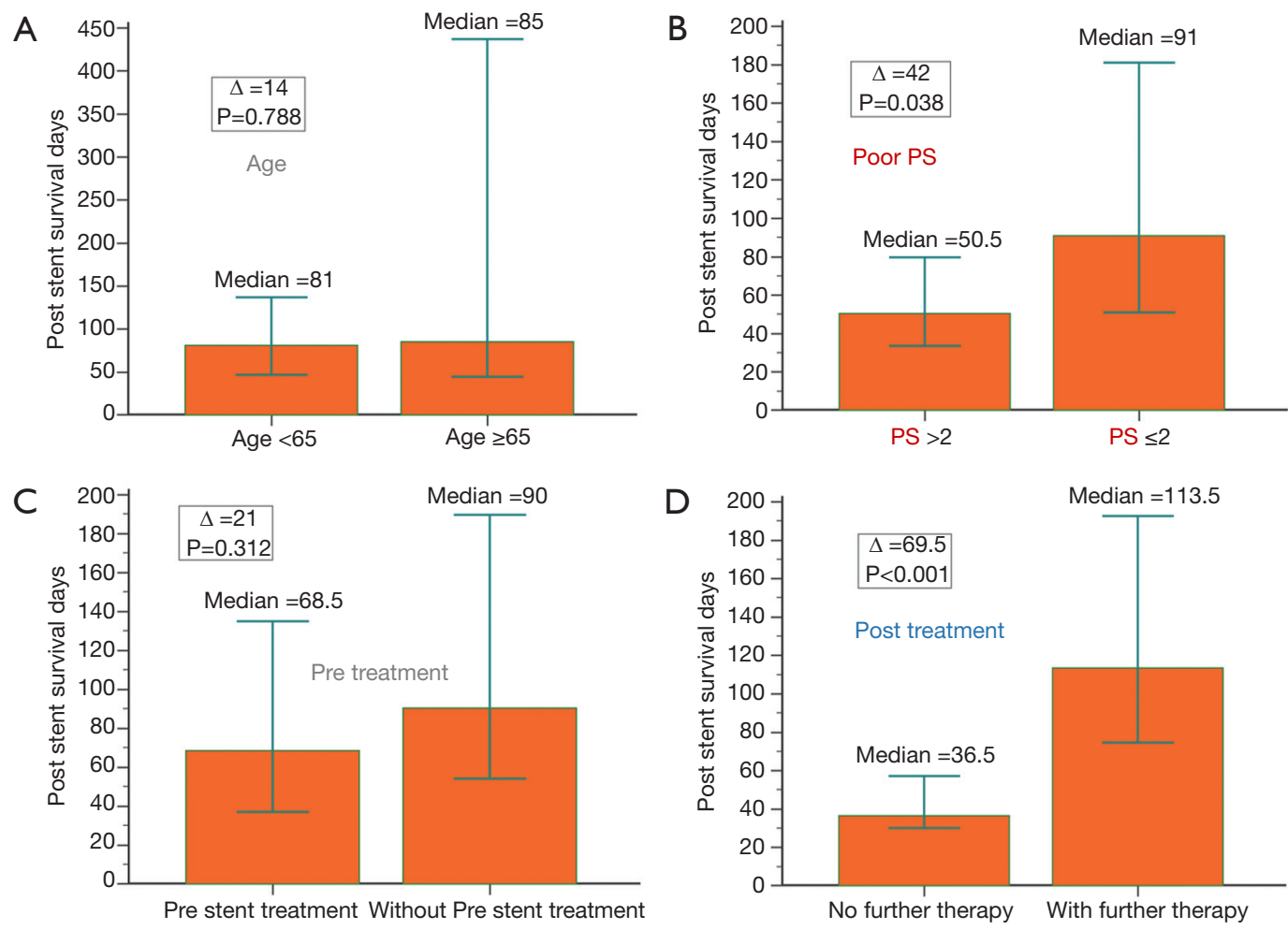

Figure 3 The median survival days after airway stenting in patients with advanced esophageal cancer and airway involvement can be predicted based on clinical variables. (A) The patients with age above 65 years had a similar survival (85 days) as those with age less than 65 years (81 days) $(\mathrm{P}=0.788)$; (B) the patients with $\mathrm{PS} \leq 2$ had a longer survival (91 days) than those with $\mathrm{PS}>2(50.5$ days) $(\mathrm{P}=0.038)$; $(\mathrm{C})$ the patients without anti-cancer treatment before airway stenting had a longer survival (90 days) than those with anti-cancer treatment (68.5 days) ( $\mathrm{P}=0.312)$; (D) a longer survival was also seen in the patients who received additional therapy (113.5 days) after airway stenting than those without further therapy (36.5 days) $(\mathrm{P}<0.001)$. PS, performance status.

Persistent pneumonia worsens survival even after successful airway stent implantation (13). In the current study, more patients who did not receive further treatment had persistent pneumonia than those who did receive further treatment (64.2\% vs. $28.6 \%)$.

$\mathrm{ARF}$ is one of the most severe complications in patients with obstruction of the trachea and main stem bronchi due to tumor invasion, external compression or TEF. In the current study, ARF before interventional bronchoscopy was an independent predictor of a poor outcome. In addition, we found that the patients with a residual tracheal diameter $<0.77 \mathrm{~cm}$ were at high risk of ARF. Moreover, more patients with ARF had persistent pneumonia than those without ARF (64.2\% vs. 28.6\%). Severe pneumonia has been 

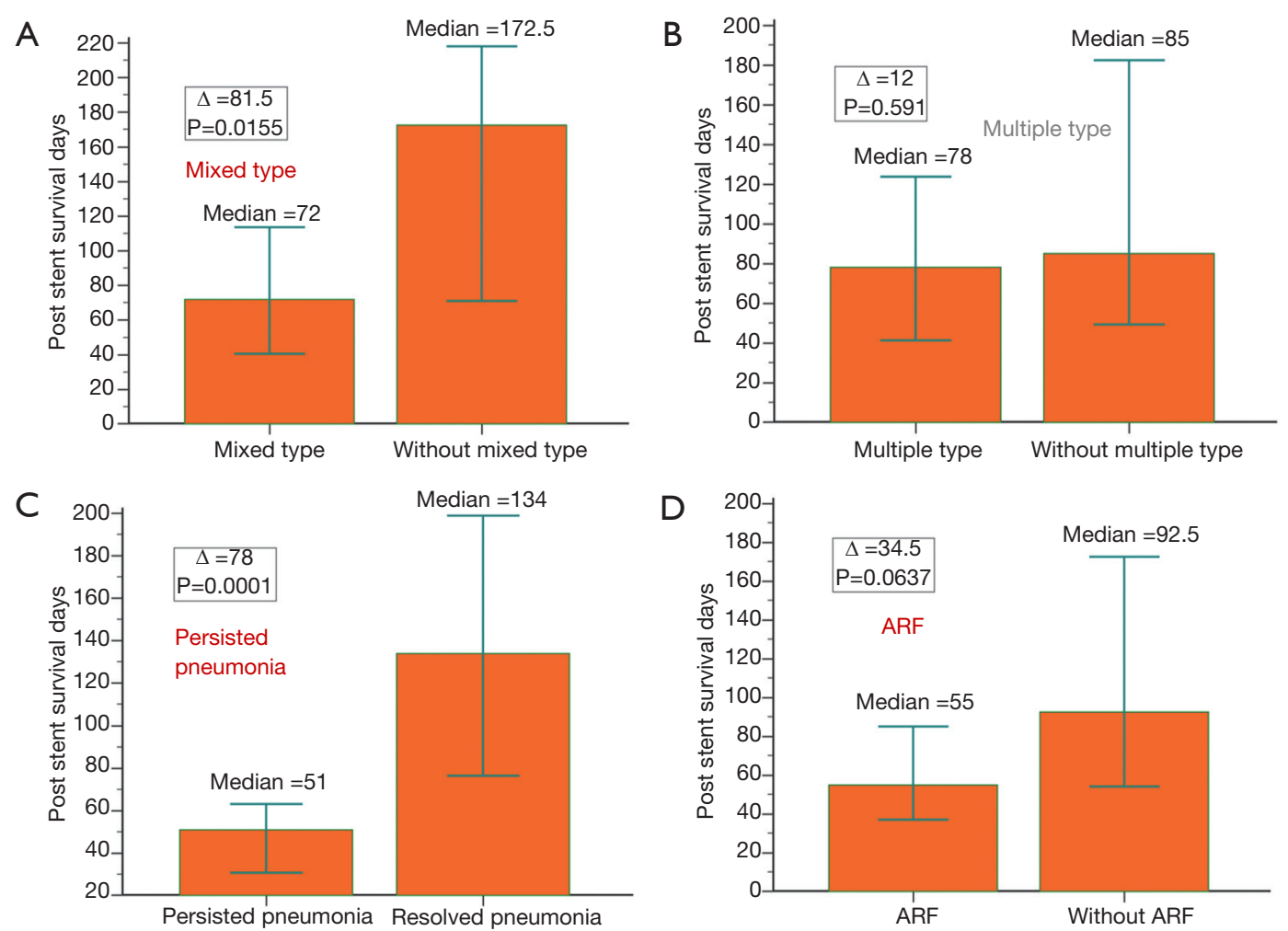

Figure 4 The median survival days after airway stenting in patients with advanced esophageal cancer and airway involvement can be predicted based on clinical variables. (A) The patients with mixed type airway involvement seemed to have a shorter survival (72 days) than those without mixed type airway involvement (172.5 days) ( $\mathrm{P}=0.015)$; (B) however, the patients with multiple type airway involvement by esophageal cancer had a similar survival (78 days) as those without multiple type airway involvement ( 85 days) $(\mathrm{P}=0.591)$; (C) in addition, the patients with persistent pneumonia (51 days) also had a worse prognosis than those without persistent pneumonia $(134$ days $)(P<0.001)$; (D) a shorter survival was also observed in the patients with $\mathrm{ARF}$ (55 days) compared to those without $\mathrm{ARF}$ (92.5 days) ( $\mathrm{P}=0.064)$. ARF, acute respiratory failure.

Table 6 The prognostic predictors of patients with airway involvement due to advanced esophageal cancer after metallic airway stenting using flexible bronchoscopy

\begin{tabular}{|c|c|c|c|c|c|c|}
\hline Variables & \multicolumn{3}{|c|}{ Univariate } & \multicolumn{3}{|c|}{ Multivariate } \\
\hline Age $>65$ & 0.42 & $0.09-1.81$ & 0.244 & - & - & - \\
\hline $\mathrm{PS}>2$ & 3.15 & $1.34-7.41$ & 0.008 & - & - & - \\
\hline Multiple site & 1.48 & $0.68-3.19$ & 0.336 & - & - & - \\
\hline Pre-stent treatment & 1.39 & $0.73-2.63$ & 0.316 & 3.429 & $1.54-7.60$ & 0.002 \\
\hline Post-stent treatment & 0.17 & $0.08-0.37$ & $<0.001$ & 0.146 & $0.06-0.34$ & $<0.001$ \\
\hline Persisted pneumonia & 5.77 & $2.57-12.90$ & $<0.001$ & - & - & - \\
\hline ARF & 2.10 & $1.062-4.156$ & 0.0327 & 5.224 & $2.23-12.26$ & $<0.001$ \\
\hline
\end{tabular}

$\mathrm{HR}$, hazard ratio; $\mathrm{Cl}$, confidence interval; PS, performance status; ARF, acute respiratory failure. 
A

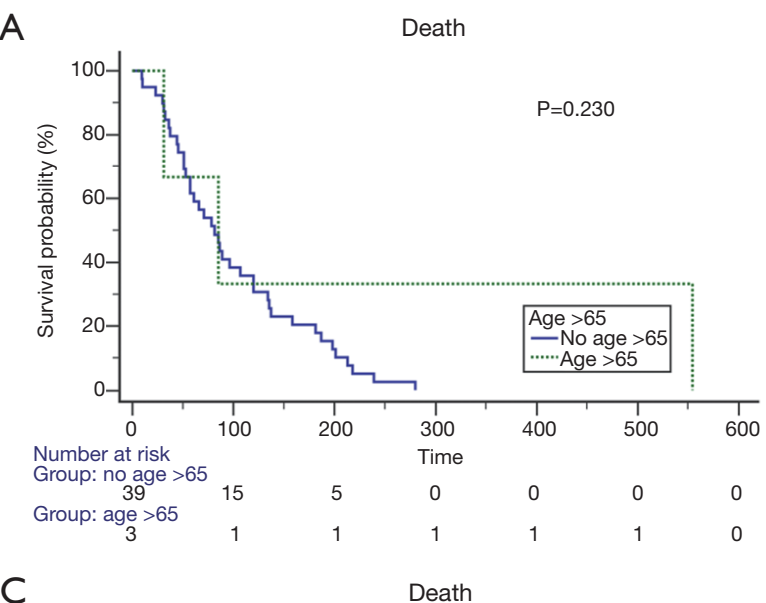

C

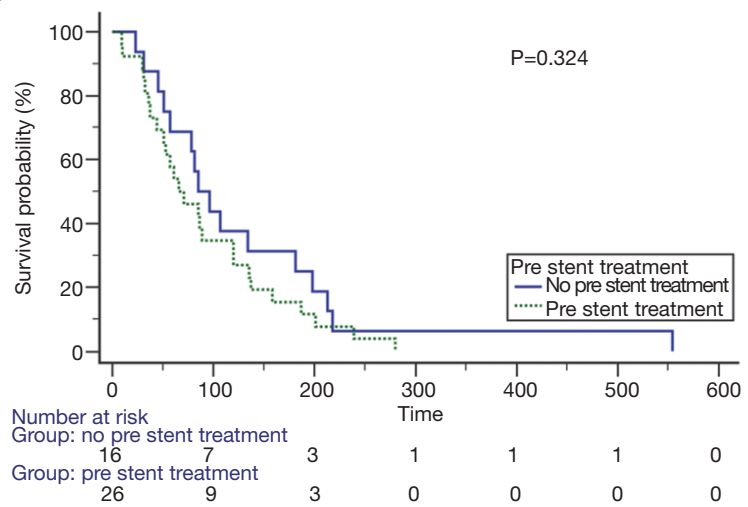

B

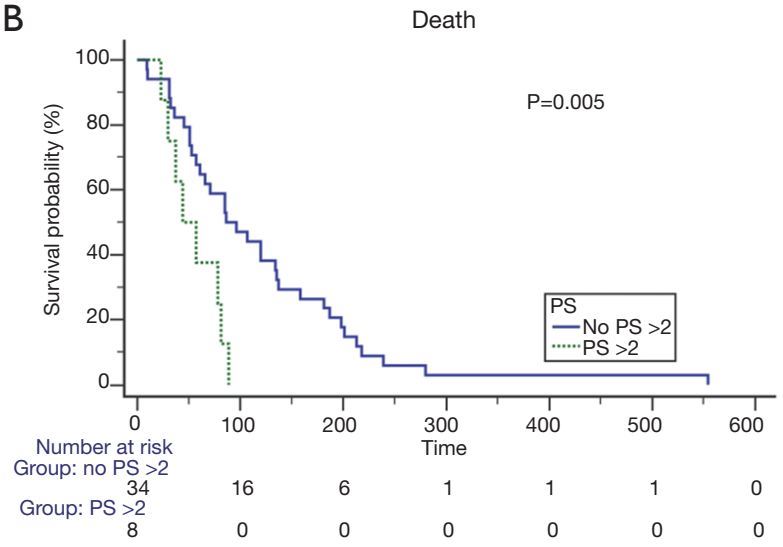

D

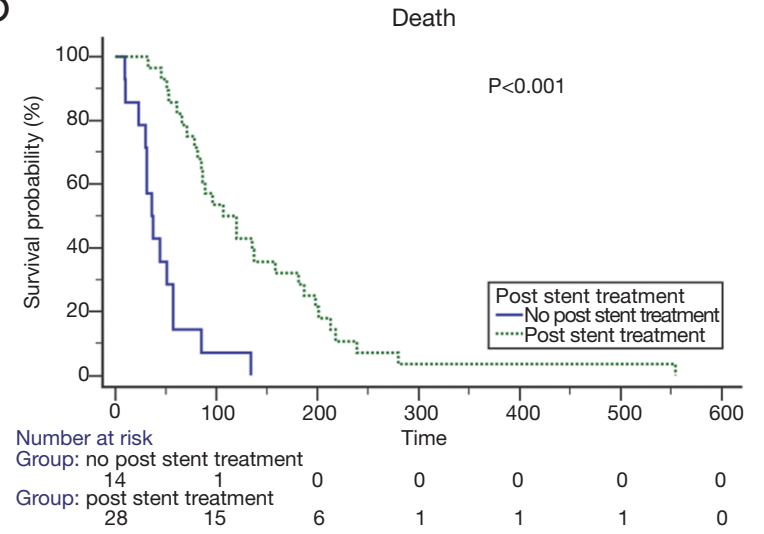

Figure 5 The Kaplan-Meier log-rank analysis was performed with all variables that may be related to survival. (A) The patients with age above 65 years had a similar survival as those with age less than 65 years $(\mathrm{P}=0.230)$; (B) the patients with $\mathrm{PS} \leq 2$ had a longer survival than those with PS >2 ( $\mathrm{P}=0.005)$; (C) the patients without anti-cancer treatment before airway stenting had a similar survival as those with anticancer treatment $(\mathrm{P}=0.324)$; (D) a longer survival was also seen in the patients who received additional therapy after airway stenting than those without further therapy $(\mathrm{P}<0.001)$. PS, performance status.

reported to be the primary factor in failure to liberate from MV after airway stenting (22). Pneumonia is a common complication in patients with CAO due to malignancy, and persistent pneumonia may be due to inadequate drainage of secretions. Airway stenting has been shown to facilitate extubation in patients with malignant $\mathrm{CAO}$, which plays a role as a bridge to additional therapy, especially in cancer treatment-naïve patients and those without severe pneumonia $(22,23)$.

The location of the fistula site caused by esophageal or lung cancer was identified as an independent risk factor predicting survival. A previous study showed that airway stenting in the RMB was associated with a significantly worse survival than airway stenting in the LMB, and that tracheal airway stenting resulted in better survival than distal airway stenting (14). In contrast, Breitenbücher et al. reported that stent insertion at a distal site resulted in significantly better survival than stent insertion at a proximal site (24). Among the 60 patients in their study, 50 had bronchial carcinoma, and bronchial carcinoma with tracheal involvement would reflect more severe disease. In our study, survival did not seem to be influenced by the location of airway involvement, and there was no significant difference in survival between the patients with multiple sites of airway involvement and those without multiple involvement (78 vs. 85 days). In addition, the patients in our study had all kinds of airway involvement, not only fistulas. Moreover, we did not include other malignancies, and only focused on esophageal cancer.

The main types of malignant airway obstruction include endobronchial invasion, external compression, fistula formation, and mixed type obstructions. The type of airway 
A

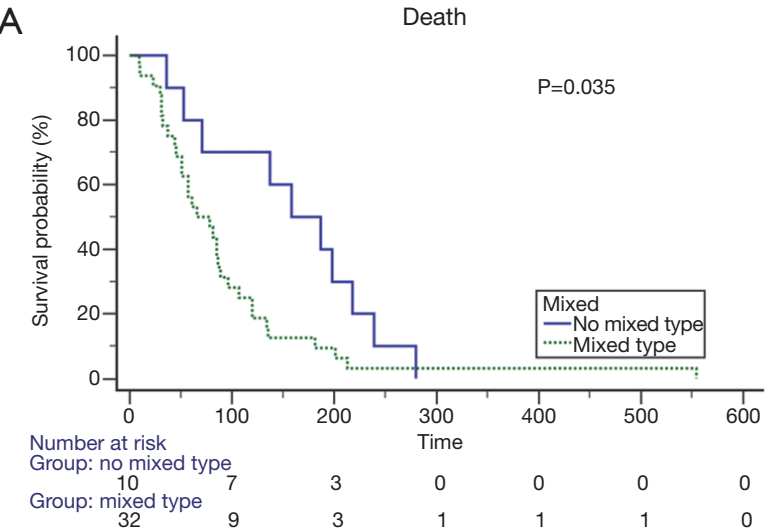

C

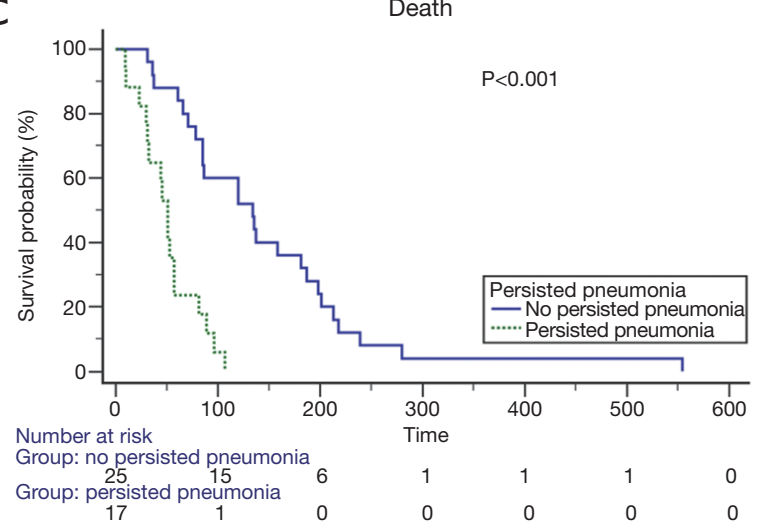

B

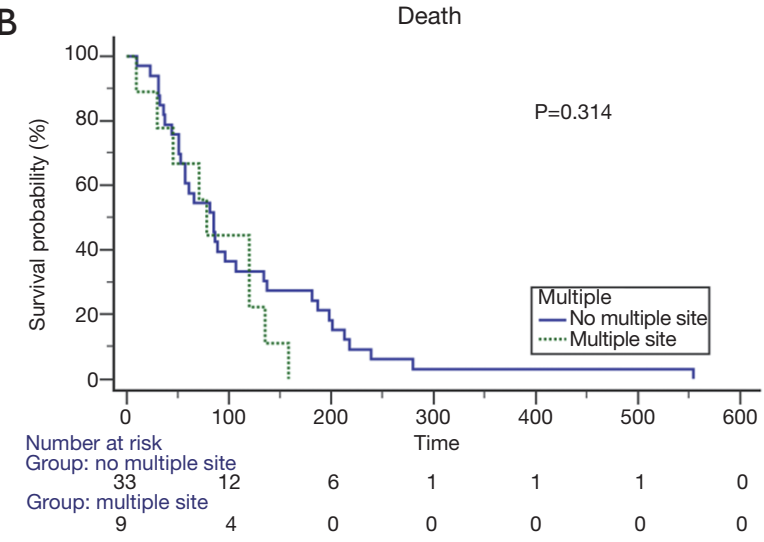

D

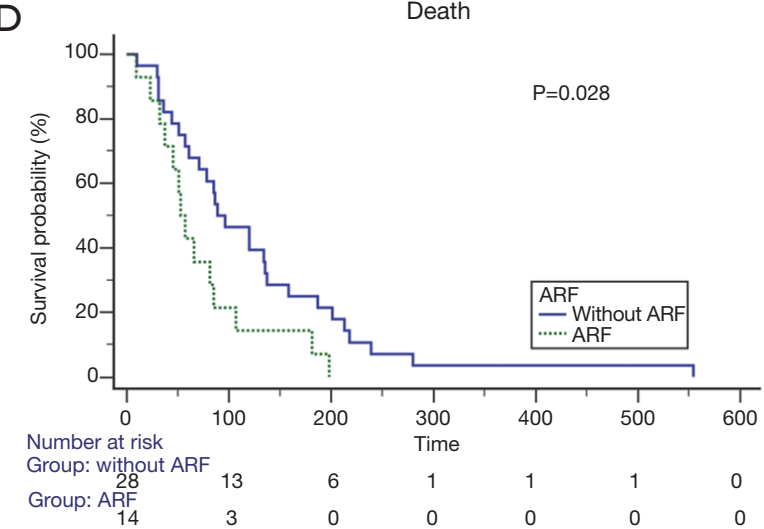

Figure 6 The Kaplan-Meier log-rank analysis was performed with all variables that may be related to survival. (A) The patients with mixed type airway involvement seemed to have a shorter survival than those without mixed type airway involvement. $(\mathrm{P}=0.035)$; (B) however, the patients with multiple type airway involvement by esophageal cancer had a similar survival as those without multiple type airway involvement $(\mathrm{P}=0.314) ;(\mathrm{C})$ in addition, the patients with persistent pneumonia also had a worse prognosis than those without persistent pneumonia $(\mathrm{P}<0.001)$; (D) a shorter survival was also observed in the patients with ARF compared to those without $A R F(P=0.028)$. ARF, acute respiratory failure.

involvement seemed to affect survival in our study, and the patients with mixed types of airway involvement had a shorter survival than those without mixed types of airway involvement (72 vs. 172.5 days). If airway obstruction is totally due to external compression caused by esophageal cancer, the only option is stent placement (25). For mixed type airway involvement, ablative therapy (laser, electrocautery, argon plasma coagulation, cryotherapy) is usually required before stent insertion, and this may impact the effectiveness.

In the current study, $52.3 \%$ of the patients had postprocedural complications, including a mucus plug in $35.9 \%$, granulation tissue in $7 \%$, and tumor ingrowth in $38.1 \%$. Miyazawa $e t a l$. reported mucus plugging in $9 \%$ of patients after the implantation of uncovered Ultraflex ${ }^{\mathrm{TM}}$ stents for malignant airway stenosis (26). This is different to our findings. We expected that covered self-expandable metal stents would have a higher risk of mucus plugging, as a higher rate of mucus plugging in was reported in $19-38 \%$ of patients after SEMS (Wallstents) implantation by Bolliger et al. (27) The complication of granulation tissue was seen in $7.1 \%$ of our patients, compared to $10-15 \%$ of the patients after Wallstents insertion in Bolliger et al.'s study (27). The main difference between covered Wallstents and Ultraflex ${ }^{\mathrm{TM}}$ stents is that the former had sharp fibers at the end of the stent which could stimulate the formation of granulation tissue. Tumor ingrowth in the stents was seen in $38.1 \%$ of our patients, which is higher compared to previous studies $(5-24 \%)(24,26)$. The exact reasons remain unclear.

There are several limitations to this study. First, this was a retrospective study with a small number patients at a single institute. Selection bias was possible, however, 
it is not easy to perform blinded, prospective controlled studies in these patients due to their critical condition of airway obstruction requiring emergency bronchoscopy interventions. Second, we did not include other objective measurements such as quality of life or pulmonary function tests of clinical improvement after the procedure. However, it is also not easy to perform pulmonary function tests in this critical scenario. Third, not all clinical variables, such as tumor-node-metastasis (TNM) staging, tumor size, decreasing body mass index (BMI), and dysphagia that may be related to survival were included in multivariate analysis due to the small number of patients. Therefore, only four potential variables (ARF, mixed type, pre-stent treatment, post-stent treatment) that may be related to survival were selected in multivariate analysis after univariate analysis. Fourth, silicone $\mathrm{Y}$ stenting with rigid bronchoscopy is considered as a standard stenting technique for patients with airway stenosis around carina. Besides, rigid bronchoscopy was a more safe and effective modality for patients with TEF. However, rigid bronchoscopy and silicone $\mathrm{Y}$ stent were not all available in our clinical practice. Fifth, the patients with TEF received airway stenting alone, single stent insertion may be insufficient for the patients with TEF. The National Comprehensive Cancer Network (NCCN) guideline recommend double stenting for patients with TEF, the airway stent should be placed first followed by the esophageal stent, preferably in one setting, although one procedure is not always feasible; this can reduce the risk of airway obstruction by the esophageal stent. However, parallel stenting should be performed after completely reviewing the patient's clinical indication because mechanical friction between the parallel stents may cause pressure necrosis of the tissue, which resulting in a fatal bleeding $(28,29)$. In our real world, the patient must afford the cost of esophageal stent, however, the cost of airway stent can be supported by Taiwan's National Health Insurance. Most patients in our study with a low socioeconomic status cannot afford the cost of double stenting, which was the reason that there were no patients who received double stenting in current study. Despite these limitations, this study is unique in that we offer several clinical predictors in patients with all forms of airway involvement due to advanced esophageal cancer after airway stenting.

\section{Conclusions}

The present study showed that metallic airway stenting with flexible bronchoscopy without fluoroscopic guidance in patients with airway involvement due to advanced esophageal cancer was safe and feasible. The positive impact on survival after airway stenting was that the patients could receive additional anti-cancer therapy, whereas previous anti-cancer treatment and ARF before interventional bronchoscopy were negative predictors for survival after airway stenting. The patients with a residual diameter of an obstructed trachea $<0.77 \mathrm{~cm}$ had a high risk of ARF. We believe that this study can help clinicians to select adequate candidates before performing interventional bronchoscopy.

\section{Acknowledgments}

None.

\section{Footnote}

Conflicts of Interest: The authors have no conflicts of interest to declare.

Ethical Statement: The authors are accountable for all aspects of the work in ensuring that questions related to the accuracy or integrity of any part of the work are appropriately investigated and resolved. This study was approved by the China Medical University Hospital Internal Review Board (DMR98-IRB-335), and the requirement for informed consent was waived.

\section{References}

1. Enzinger PC, Mayer RJ. Esophageal cancer. N Engl J Med 2003;349:2241-52.

2. Murthy S, Gonzalez-Stawinski GV, Rozas MS, et al. Palliation of malignant aerodigestive fistulae with selfexpanding metallic stents. Dis Esophagus 2007;20:386-9.

3. Sihoe AD, Wan IY, Yim AP. Airway stenting for unresectable esophageal cancer. Surg Oncol 2004;13:17-25.

4. Amjadi K, Voduc N, Cruysberghs Y, et al. Impact of interventional bronchoscopy on quality of life in malignant airway obstruction. Respiration 2008;76:421-8.

5. Wood DE. Management of malignant tracheobronchial obstruction. Surg Clin North Am 2002;82:621-42.

6. Ost DE, Ernst A, Grosu HB, et al. Therapeutic bronchoscopy for malignant central airway obstruction: success rates and impact on dyspnea and quality of life. Chest 2015;147:1282-98.

7. Jeon K, Kim H, Yu CM, et al. Rigid bronchoscopic 
intervention in patients with respiratory failure caused by malignant central airway obstruction. J Thorac Oncol 2006;1:319-23.

8. Chen CH, Wu BR, Cheng WC, et al. Interventional pulmonology for patients with central airway obstruction: an 8-year institutional experience. Medicine (Baltimore) 2017;96:e5612.

9. Shin JH, Song HY, Ko GY, et al. Esophagorespiratory fistula: long-term results of palliative treatment with covered expandable metallic stents in 61 patients. Radiology 2004;232:252-9.

10. Li YD, Li MH, Han XW, et al. Gastrotracheal and gastrobronchial fistulas: management with covered expandable metallic stents. J Vasc Interv Radiol 2006; 17:1649-56.

11. Shaffer JP, Allen JN. The use of expandable metal stents to facilitate extubation in patients with large airway obstruction. Chest 1998;114:1378-82.

12. Venuta F, Rendina EA, De Giacomo T, et al. Endoscopic treatment of lung cancer invading the airway before induction chemotherapy and surgical resection. Eur J Cardiothorac Surg 2001;20:464-7.

13. Chung FT, Lin HC, Chou CL, et al. Airway Ultraflex stenting in esophageal cancer with esophagorespiratory fistula. Am J Med Sci 2012;344:105-9.

14. Herth FJ, Peter S, Baty F, et al. Combined airway and oesophageal stenting in malignant airway-oesophageal fistulas: a prospective study. Eur Respir J 2010;36:1370-4.

15. Chhajed PN, Somandin S, Baty F, et al. Therapeutic bronchoscopy for malignant airway stenoses: choice of modality and survival. J Cancer Res Ther 2010;6:204-9.

16. Hara H, Kuwano K, Mori S. Recurrent and persistent pneumonia. Eur J Intern Med 2018;53:e8-9.

17. Saji H, Furukawa K, Tsutsui H, et al. Outcomes of airway stenting for advanced lung cancer with central airway obstruction. Interact Cardiovasc Thorac Surg 2010;11:425-8.

18. Song JU, Park HY, Kim H, et al. Prognostic factors for bronchoscopic intervention in advanced lung or esophageal cancer patients with malignant airway obstruction. Ann

Cite this article as: Cheng $\mathrm{WC}$, Shen $\mathrm{MF}, \mathrm{Wu} \mathrm{BR}$, Chen CY, Chen WC, Liao WC, Chen CH, Tu CY. The prognostic predictors of patients with airway involvement due to advanced esophageal cancer after metallic airway stenting using flexible bronchoscopy. J Thorac Dis 2019;11(9):3929-3940. doi: 10.21037/ jtd.2019.08.108
Thorac Med 2013;8:86-92.

19. McGrath EE, Warriner D, Anderson P. The insertion of self expanding metal stents with flexible bronchoscopy under sedation for malignant tracheobronchial stenosis: a single-center retrospective analysis. Arch Bronconeumol 2012;48:43-8.

20. Ahn Y, Chang H, Lim YS, et al. Primary tracheal tumors: review of 37 cases. J Thorac Oncol 2009;4:635-8.

21. Nagano H, Kishaba T, Nei Y, Yamashiro S, Takara $H$. Indications of airway stenting for severe central airway obstruction due to advanced cancer. PLoS One 2017;12:e0179795.

22. Lin SM, Lin TY, Chou CL, et al. Metallic stent and flexible bronchoscopy without fluoroscopy for acute respiratory failure. Eur Respir J 2008;31:1019-23.

23. Oki M, Saka H, Hori K. Airway stenting in patients requiring intubation due to malignant airway stenosis: a 10-year experience. J Thorac Dis 2017;9:3154-60.

24. Breitenbücher A, Chhajed PN, Brutsche MH, et al. Longterm follow-up and survival after Ultraflex stent insertion in the management of complex malignant airway stenoses. Respiration 2008;75:443-9.

25. Ernst A, Silvestri GA, Johnstone D, et al. Interventional pulmonary procedures: guidelines from the American College of Chest Physicians. Chest 2003;123:1693-717.

26. Miyazawa T, Yamakido $M$, Ikeda S, et al. Implantation of Ultraflex nitinol stents in malignant tracheobronchial stenoses. Chest 2000;118:959-65.

27. Bolliger CT, Heitz M, Hauser R, et al. An airway Wallstent for the treatment of tracheobronchial malignancies. Thorax 1996;51:1127-9.

28. Kishi K, Nakao T, Goto H, et al. A fast placement technique for covered tracheobronchial stents in patients with complicated esophagorespiratory fistulas. Cardiovasc Intervent Radiol 2005;28:485-9.

29. Nam DH, Shin JH, Song HY, Jung GS, Han YM. Malignant esophageal-tracheobronchial strictures: parallel placement of covered retrievable expandable nitinol stents. Acta Radiol 2006;47:3-9. 